Check for updates

Cite this: RSC Adv., 2017, 7, 54117

\title{
Recycling and reuse of waste artificial turf via solid- state shear milling technology
}

\author{
Qing Liu, (D) a Pan He, (D) a Shuangqiao Yang, (D) ${ }^{a}$ Shibing Bai (D) *a \\ and Wenfeng Duan (D) ${ }^{\mathrm{b}}$
}

Nowadays, the amount of the artificial turf waste is very huge, as it has been applied widely. However, it is a great challenge to recycle and reuse waste artificial turf (WAT) because its components are complex and hard to separate. In this study, solid-state shear milling $\left(S^{3} M\right)$ technology was introduced to recycle WAT into composite powder with valuable properties through ultrafine milling of WAT at room temperature. The results showed that the WAT included 46.7 wt\% PE, 8.7 wt\% PP, 2.4 wt\% PET, 16.1 wt\% polyacrylate and 26.1 wt\% inorganic infill. The results of SEM and ultra-depth three-dimensional (3D) microscopy indicated that the domain size of the recycled material became smaller with the increase of milling cycles. The domain size and the performance of the recycled material could be controlled by the particle size and the size distribution of the composite powder in the solid state. The dynamic mechanical analysis (DMA) and $\mathrm{X}$-ray diffraction (XRD) patterns demonstrated that the $S^{3} \mathrm{M}$ technology improved the compatibility between the components and effectively decreased the crystallite size of PE in WAT. The melt flow index and the high capillary rheology test indicated that the thermal processability of the recycling material became better through $\mathrm{S}^{3} \mathrm{M}$ milling. The tensile strength and the elongation at break of the recycling material reached $12.6 \mathrm{MPa}$ and $96.3 \%$, respectively. What's more, low-price wood-plastic products with good performance and appearance could be manufactured using the recycled materials.

Received 11th October 2017

Accepted 17th November 2017

DOI: 10.1039/c7ra11206h

rsc.li/rsc-advances
Currently, the main disposal ways ${ }^{1,3}$ for WAT include landfill, incineration and respectively recycling after complete separating the ingredients. Both landfilling and incineration are the most unwelcome method, because landfilling occupies a large land space and contaminates groundwater ${ }^{3-7}$ and incinerating WAT releases a substantial number of poisonous gases. Separating the WATs' ingredients and recycling the parts is a common method, ${ }^{3}$ but this disposal way presented ultra-low recycling efficiency owing to WATs' complex and difficult-toseparate constituents. Cheng ${ }^{3}$ suggested that the WAT could be shredded, repalletized and converted into usable materials for new artificial turf or other extruded plastic products. However, the products have poor mechanical properties and only use for lower grade applications, ${ }^{1}$ limiting its wide application. Thus, it is vital to develop new methods for recycling WAT and turning the wasters into usable products.

The solid-state shear milling equipment was invented in our laboratory, ${ }^{8-12}$ whose schematic diagrams of the solid state shear milling reactor and the inlaid pan-milling are shown in Fig. 1 as following. The main structural parameters of the panmill are: radius $R$, division number $n$, slot number in each $m$, bevel angle $\alpha$ and slot top with $\delta$. Theoretical analysis demonstrates that this equipment has excellent pulverizing and dispersing effects on polymeric materials owing to the ingenious design derived from the traditional Chinese stone-mill. The key part of this innovation equipment is the inlaid pan,
${ }^{a}$ State Key Laboratory of Polymer Materials Engineering, Polymer Research Institute of Sichuan University, Chengdu 610065, China. E-mail: baishibing@scu.edu.cn; Tel: +86-028-85463909

${ }^{b}$ State Key Laboratory of Special Functional Waterproof Materials, Beijing Oriental Yuhong Waterproof Technology Co., Ltd, Beijing 100000, China 


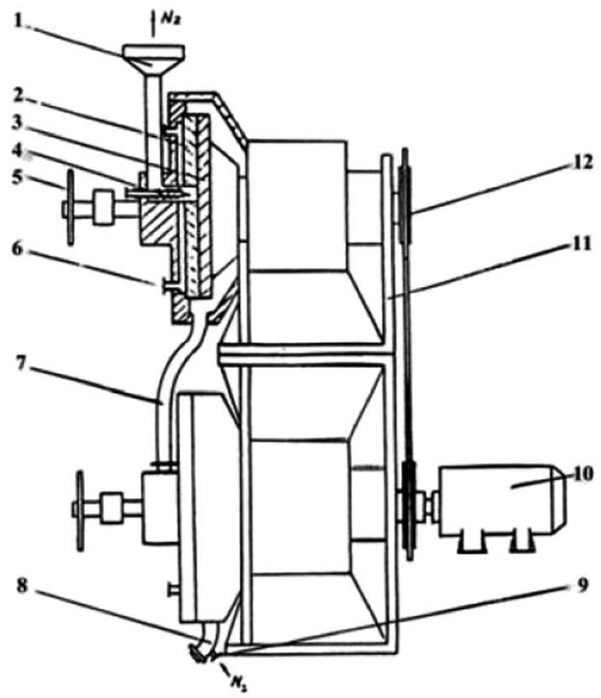

(a)

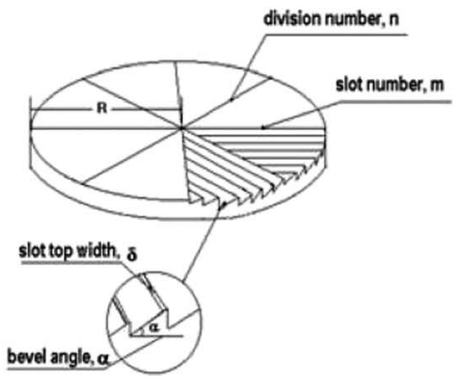

(b)

Fig. 1 Schematic diagrams of (a) solid state shear milling reactor and (b) inlaid mill-pan (note: (1) inlet; (2) stationary; (3) moving pan; (4) feeding screw; (5) handle; (6) medium entrance; (7) flexible tube; (8) outlet; (9) entrance of inert gas; (10) stand; (12) drive system).

whose surface is divided into equal sectors by several bevels; the ridges of the bevels were parallel with the dividing lines, as shown in Fig. 1b. Functioning like a pair of three-dimensional scissors provides very strong shear forces for pulverization, dispersion, mixing and activation. Previous studies showed that state shear milling $\left(\mathrm{S}^{3} \mathrm{M}\right)$ technology can be used in producing ultrafine polymer powders, ${ }^{\mathbf{8} 10,11}$ tire rubbers decrosslinking, ${ }^{13}$ carbon nano-tube nanoscale cutting, ${ }^{14}$ montmorillonite nanoscale exfoliation ${ }^{15}$ and graphene nanoscale dispersion. ${ }^{9}$ Recently, it was found that $\mathrm{S}^{3} \mathrm{M}$ technology may show a potential application for recycling waste polymers with immiscible and complex components into value-added products without sorting by types or color. ${ }^{10,11}$ As compared with other technologies, etc., landfill, incineration and other recycling methods, the new recycling method doesn't need not to require efforts to handle and separate WATs' ingredients. The solid state shear milling reactor adopted its three-dimensional shear to ultrafine mill WAT at room temperature, only by ultrafine milling WAT at room temperature, the domain size and the performance of the material were controlled by the particle sizes and its distribution of the composite powder at the solid state.

In this study, the $\mathrm{S}^{3} \mathrm{M}$ technology was used to recycle WAT to develop a new recycling method for WAT. The morphology, compatibility, crystalline structure, processabilities and mechanical properties of the recycled WAT were investigated. What's more, the WAT fine powder was used to replace polyethylene materials to prepare wood-plastics products.

\section{Experimental}

\subsection{Materials}

The WAT, which were used, were provided by Nanjing Jufeng Advanced Materials Co., Ltd. The high-density polyethylene (HDPE) is a commercially available material from SINOPEC
Maoming Company, Guangdong, China (production code $14101906422 \mathrm{C}$ TR480M), $\mathrm{MI}=0.5 \mathrm{~g} / 10 \min \left(210^{\circ} \mathrm{C}, 2.16 \mathrm{~kg}\right)$, density $=0.944 \mathrm{~g} \mathrm{~cm}^{-3}\left(23{ }^{\circ} \mathrm{C}\right)$. The wood powders (WPs) (average size $47 \mathrm{~mm}$ ) used in this study were purchased from Jiangsu biomass material Co., Ltd. China.

\subsection{Preparation of WAT recycling material and wood- plastics composites}

WAT was disposed directly (without cleaning) and integrally in accordance with the following process. The WAT fragments were firstly smashed by plastic crusher (PC400, Jinshi Plastic Machinery of Chao'an, China) and then fed into the pan-mill through the hopper in the center of the milling pan at a rotation speed of $20 \mathrm{rpm}$. The milled materials were discharged from the brim of the pan, and the discharged powder was collected for the next milling cycle. The heat was removed by the circulating water. Sampling $500 \mathrm{~g}$ powder milled 5 cycles, 10 cycles, 15 cycles, 20 cycles, successively and respectively. And contrastively, $500 \mathrm{~g}$ unmilled WAT fragment were sampled.

The prepared powders (and fragments) were extruded into threads with a twin-screw extruder (SHJ-20, $\Phi=25 \mathrm{~mm}, L / D=$ 33, Nanjing Giant Machinery Co. Ltd., China), the temperatures of barrels were $170{ }^{\circ} \mathrm{C}, 190{ }^{\circ} \mathrm{C}, 190{ }^{\circ} \mathrm{C}, 190{ }^{\circ} \mathrm{C}$, and $185^{\circ} \mathrm{C}$ and the screw was maintained at a speed of $120 \mathrm{rpm}$. Eventually the standard test specimens were obtained by means of the injection-molding machine (MA1200/370, Haitian Plastic Machinery Co. Ltd., China) under the conditions of $190{ }^{\circ} \mathrm{C}$. Signing the products prepared by WAT fine powders as r-WAT.

The samples which were used for WAT components analysis were cut into bulk sample with the shape of $45 \mathrm{~mm} \times 55 \mathrm{~mm}$, then separating the sample to get the components: the artificial grass fibers, the carpet backing and the adhesive, weighing $1.99 \mathrm{~g}, 1.80 \mathrm{~g}, 0.47 \mathrm{~g}$, respectively. 
The HDPE/WAT/WPs (20/20/60) composites and HDPE/WPs (40/60) composites were prepared by extrusion molding, and then cut into test specimens with the dimensions of $80 \mathrm{~mm} \times$ $10 \mathrm{~mm} \times 4 \mathrm{~mm}(L \times W \times T)$ for testing.

The scheme describing the full procedure was showed in Fig. 2.

\subsection{Analytical methods}

2.3.1 Fourier transform infrared (FT-IR) spectroscopy. FTIR spectras of the molecular structure were recorded on a Nicolet 6700 FT-IR spectrometer (Thermo Nicolet Ltd, Vernon Hills, IL, USA).

2.3.2 Differential scanning calorimetric (DSC) analysis. Melting temperature $\left(T_{\mathrm{m}}\right)$ of the artificial grass fibers and carpet backing were performed on a TA Q20 differential scanning calorimeter (TA Instruments, USA), using a heat rate $10{ }^{\circ} \mathrm{C} \mathrm{min}{ }^{-1}$ in nitrogen atmosphere at temperature from $40{ }^{\circ} \mathrm{C}$ to $300{ }^{\circ} \mathrm{C}$.

2.3.3 Thermal gravimetric analysis (TGA). TGA curves of the adhesive was done in a TGA-Q50 (TA Instruments Co. Ltd, New Castle, DE, USA), using a heat rate $10{ }^{\circ} \mathrm{C} \mathrm{min}{ }^{-1}$ in nitrogen atmosphere from $40{ }^{\circ} \mathrm{C}$ to $700{ }^{\circ} \mathrm{C}$.

2.3.4 Scanning electron microscopy (SEM). The morphology of liquid-nitrogen-cryofractured section of r-WAT specimens and the surface appearance of the extrudater were performed on scanning electron microscope (FEI Instrument Co. Ltd, USA).

2.3.5 Ultra-depth three-dimensional (3D) microscope. The optical images of components' morphology were taken by ultradepth three-dimensional microscope (VHX-1000C, KEYENCE, Japan) in 1100 magnification under the condition of hard light.

2.3.6 X-ray diffraction (XRD). X-ray diffraction was performed using a DX-1000 diffractometer (Dandong Fangyuan Instrument Co., Ltd, China). The $\mathrm{Cu} \mathrm{K} \alpha$ generator system was operated at $40 \mathrm{kV}$ and $25 \mathrm{~mA}$, and the scanning $2 \theta$ ranged from $5^{\circ}$ to $40^{\circ}$, with a scanning rate of $1^{\circ} \mathrm{min}^{-1}$.

2.3.7 Dynamic mechanical analysis (DMA). The dynamic mechanical properties of r-WAT specimens were studied by using a DMA of TA instruments (model Q800) in three-point bending mode. Dynamic loss $(\tan \delta)$ was determined at a frequency of $1 \mathrm{~Hz}$ and a heating rate of $3{ }^{\circ} \mathrm{C} \mathrm{min}^{-1}$ as a function of temperature in the range of $-100^{\circ} \mathrm{C}$ to $170^{\circ} \mathrm{C}$.

2.3.8 Melt flow index (MFI) measurements. The flowability of the product was investigated at a temperature of $210{ }^{\circ} \mathrm{C}$ and under a load of $5 \mathrm{~kg}$ by means of a MFI (XNR-400, Chengde Jinjian Testing Instrument Co. Ltd, China).

2.3.9 The capillary rheology test. The capillary rheology measurements were carried out on high-pressure capillary rheometer (Rosand RH7D, Malvern Instruments Co. Ltd, Britain). The sample was first fed in the barrel of the rheometer and preheated for $3 \mathrm{~min}$ at $200{ }^{\circ} \mathrm{C}$. Subsequently, the measurement was performed in the shear rate range of $50-3000 \mathrm{~s}^{-1}$ for all samples.

2.3.10 Mechanical test. The tensile strength various samples were tested by a universal testing machine (RG-L-10, Reger Instrument, Co. Ltd, China) at room temperature with a cross-head speed of $50 \mathrm{~mm} \mathrm{~min}{ }^{-1}$. The flexural properties of wood/plastics composites were tested by a universal testing machine (RG-L-10, Reger Instrument, Co. Ltd, China) at room temperature with a cross-head speed of $2 \mathrm{~mm} \mathrm{~min}^{-1}$. The shore $D$ hardness were carried out by Instron hardness tester.

\section{Results and discussion}

\subsection{Analysis of WAT components}

Fig. 3a showed the infrared absorption peak of the artificial grass fibers of WAT. The peaks at $2917 \mathrm{~cm}^{-1}$ and $2850 \mathrm{~cm}^{-1}$ are corresponded to the stretch vibration of methylene, the peak at $1467 \mathrm{~cm}^{-1}$ was assigned to the bending vibration of methylene and the bands at $719 \mathrm{~cm}^{-1}$ was due to the bending vibration of $-\left(\mathrm{CH}_{2}\right)_{n}{ }^{-}$, totally matching with spectrogram of polyethylene (PE). ${ }^{16}$ Fig. 4 a depicted the DSC melting curve of the artificial grass fibers, which shows $T_{\mathrm{m}}$ of artificial grass fibers is $127^{\circ} \mathrm{C}$, and for neat HDPE, the typical $T_{\mathrm{m}}$ value is $130^{\circ} \mathrm{C} .{ }^{17}$ Thereby, the characterization indicated that the element of the artificial grass fibers was polyethylene.

The carpet backing consists of the backing non-woven fabrics, adhering to the artificial grass fibers, and the backing

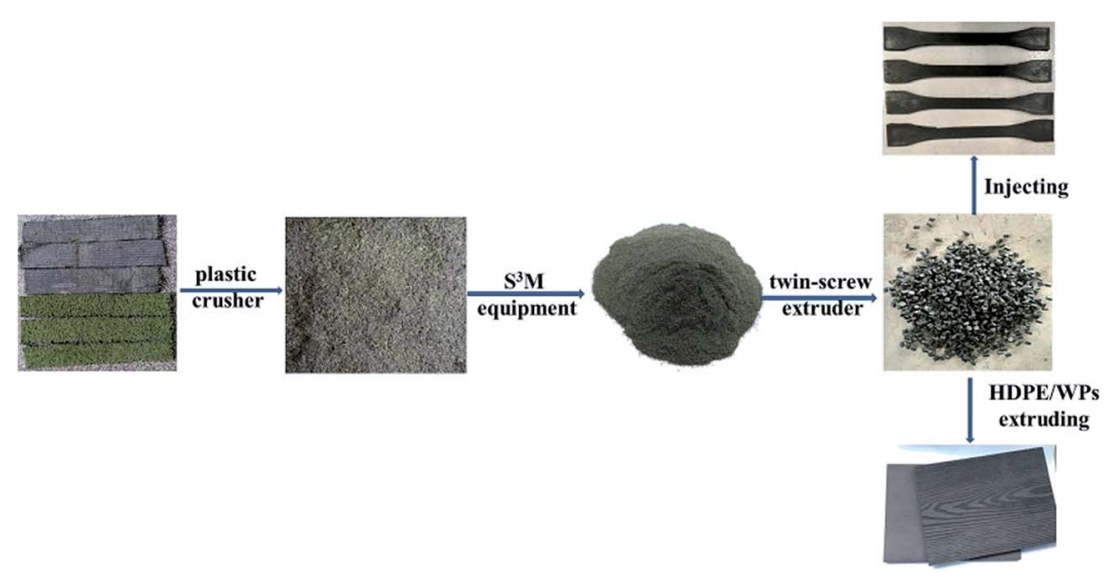

Fig. 2 The scheme describing the full procedure. 


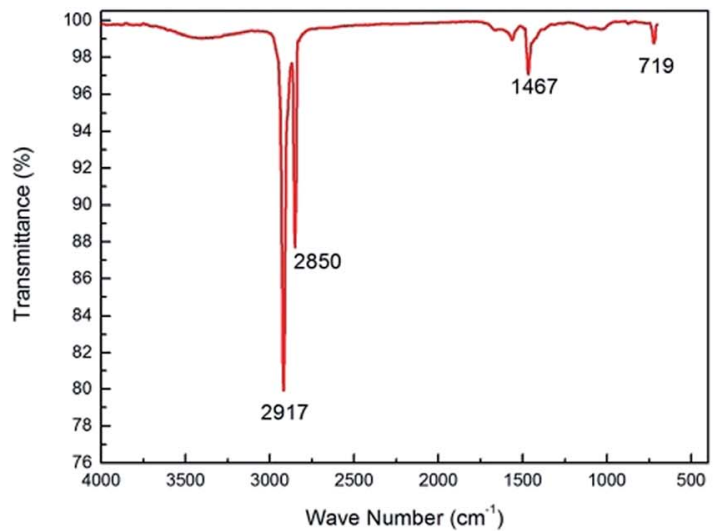

(a)

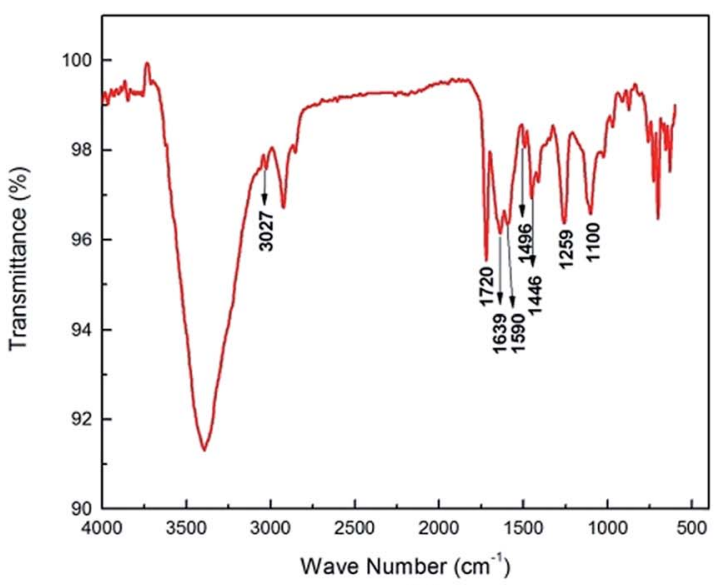

(c)

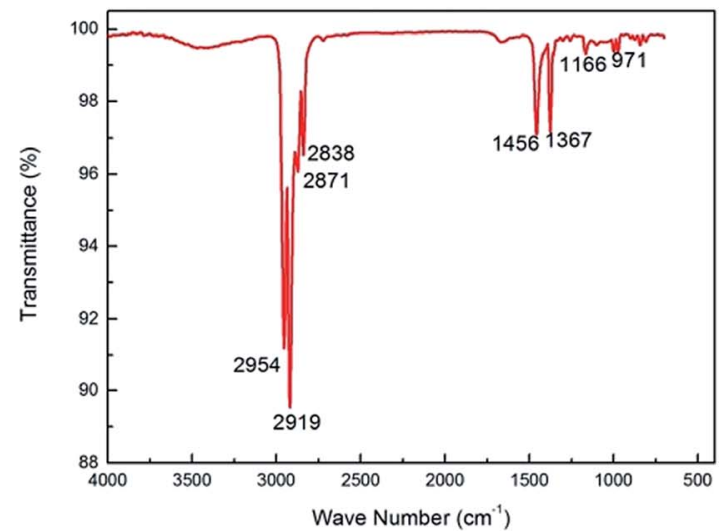

(b)

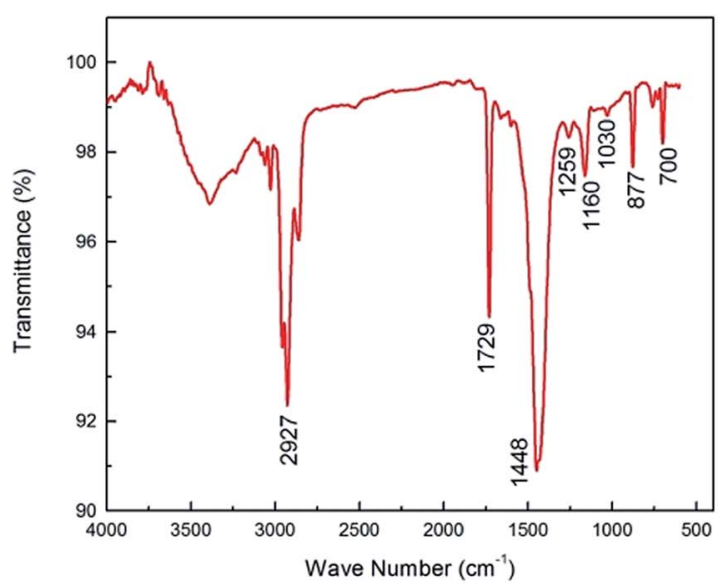

(d)

Fig. 3 FT-IR absorption spectrograms of WATC: (a) the artificial grass fibers; (b) the backing non-woven fabrics; (c) the backing felt; (d) the artificial grass adhesive.

felt which adhered to the adhesive. Fig. $3 \mathrm{~b}$ showed the infrared absorption peak of the backing non-woven fabrics. The peaks at $2954 \mathrm{~cm}^{-1}$ and $2871 \mathrm{~cm}^{-1}$ are stretching vibration of methyl, the peaks at $1367 \mathrm{~cm}^{-1}, 1166 \mathrm{~cm}^{-1}$ and $971 \mathrm{~cm}^{-1}$ are bending vibration of methyl and the peaks at $2919 \mathrm{~cm}^{-1}$ and $2838 \mathrm{~cm}^{-1}$ is stretching vibration of methylene, the peaks at $1456 \mathrm{~cm}^{-1}$ is bending vibration of methylene matched with spectrogram of polypropylene (PP). ${ }^{18}$ Fig. 3c showed the infrared absorption peak of the backing felt. The absorption peaks at $1639 \mathrm{~cm}^{-1}$, $1590 \mathrm{~cm}^{-1}, 1496 \mathrm{~cm}^{-1}$ and $1446 \mathrm{~cm}^{-1}$ are corresponding to the stretching vibration of $\mathrm{C}=\mathrm{C}$ in the benzene rings and the stretching vibration of $\mathrm{C}-\mathrm{H}$ in the benzene rings confirmed the existence of benzene which was show at $3027 \mathrm{~cm}^{-1}$. And the band at $1720 \mathrm{~cm}^{-1}, 1259 \mathrm{~cm}^{-1}$ and $1100 \mathrm{~cm}^{-1}$ were assigned to the stretch vibration of $\mathrm{C}=\mathrm{O}$ and the stretch vibration of $\mathrm{C}-\mathrm{O}-$ $\mathrm{C}$, respectively, confirming that this component is a kind of polyester. Moreover, the absorption peak fitly matching with spectrogram of polyethylene terephthalate (PET). ${ }^{19}$

Fig. $4 \mathrm{~b}$ depicted the DSC melting curve of the carpet backing, from this plot, double melting peaks were detected. The lower $T_{\mathrm{m}}$ is $169{ }^{\circ} \mathrm{C}$, which is melting temperature of $\mathrm{PP}^{20}$ and the higher $T_{\mathrm{m}}$ is $249{ }^{\circ} \mathrm{C}$, which is melting temperature of PET. ${ }^{19}$ Thereby, it is indicated that the artificial grass fibers includes polypropylene and poly(ethylene terephthalate). Besides, we weighed the backing non-woven fabrics and the backing felt respectively, noting the value $0.37 \mathrm{~g}$ and $0.10 \mathrm{~g}$. Thus, the proportion of the backing non-woven fabrics is $94.44 \%$ and the backing felt is $5.56 \%$ in the carpet backing.

Fig. $3 \mathrm{~d}$ showed the infrared absorption peak of the artificial grass adhesive. The absorption peak at $1729 \mathrm{~cm}^{-1}$ belonged to the stretch vibration of $\mathrm{C}=\mathrm{O}$, the peaks at $1259 \mathrm{~cm}^{-1}$, $1160 \mathrm{~cm}^{-1}$ and $1030 \mathrm{~cm}^{-1}$ belonged to the stretch vibration of $\mathrm{C}-\mathrm{O}-\mathrm{C}$, and the peak at $1448 \mathrm{~cm}^{-1}$ belonged to the bending vibration of methylene, which were characteristic absorption of polyacrylate. Besides, the absorption peak curve fitly matching with spectrogram of polyacrylate. ${ }^{21}$

Thermal gravimetric analysis curves of the artificial grass adhesive were depicted in Fig. 5, which showed a big falling platform proved to be the disappearance of calcium carbonate. In this work, for preparation of test samples, the adhesive was immersed and disposed using hydrochloric acid, then washed and dried sample, noting as $\mathrm{H}$-adhesive.

The weight of the sample before and after treating were $0.1225 \mathrm{~g}$ and $0.0467 \mathrm{~g}$, respectively. Thereby, it is indicated that the major components of the artificial grass adhesive are thermoset material polyacrylate and calcium carbonate inorganic infills. 


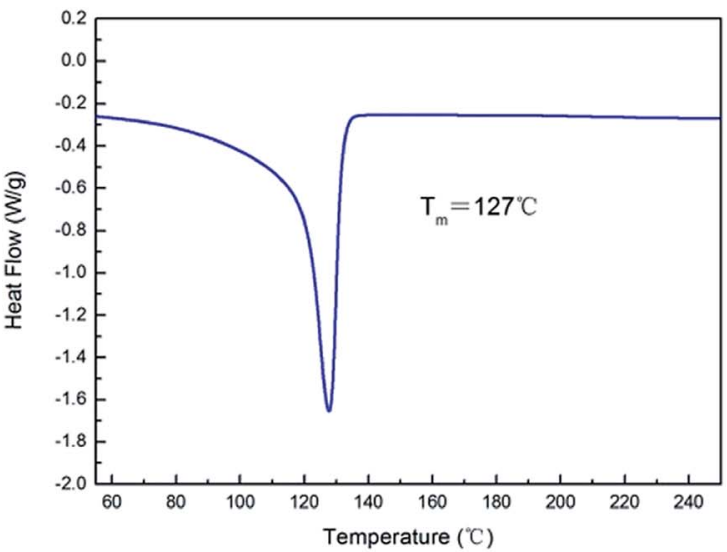

(a)

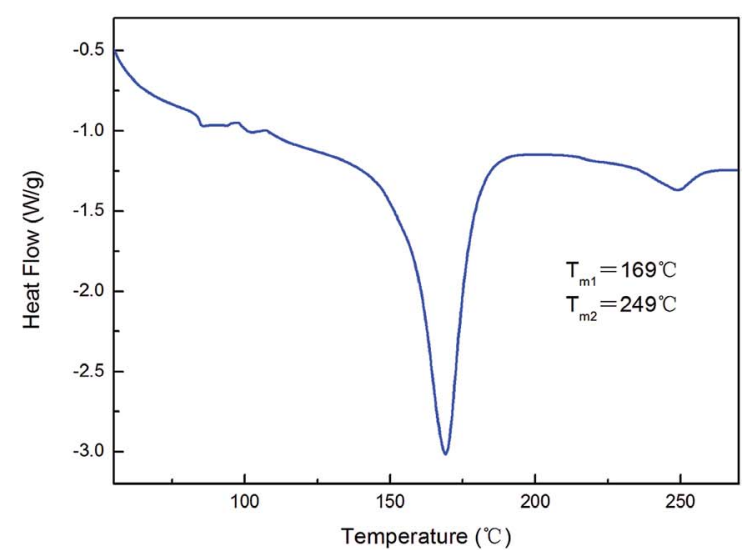

(b)

Fig. 4 DSC plots of the artificial grass fibers (a) and the carpet backing (b).

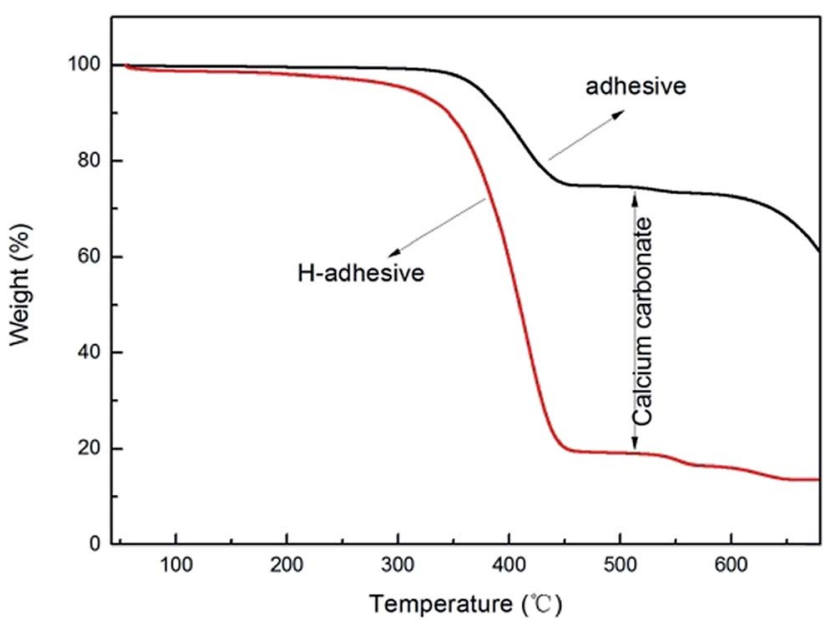

Fig. 5 TGA plots of the artificial grass adhesive.

To sum up, the components of WAT is $46.7 \% \mathrm{PE}, 8.7 \% \mathrm{PP}$, $2.4 \%$ PET, $16.1 \%$ polyacrylate and $26.1 \%$ inorganic infills.

\subsection{Morphology and compatibility}

According to the above analysis, the main ingredient of r-WAT materials prepared by indiscriminate fine WAT powders was $\mathrm{PE}$ and other components phases dispersed in PE phases. Morphology control of the dispersed phase is extremely important to achieve good properties for the multicomponent.

Fig. 6 clearly showed that the size of cavities existing in continuous phase gets smaller and the distribution are betterproportioned with the increasing milling cycles. The component analysis, mentioned above, told us that there are $26.1 \%$ calcium carbonate infills in WAT. In this section, the liquidnitrogen-cryofractured surface of r-WAT specimens have been etched by hydrochloric acid before being sputter-coated with gold. The r-WAT specimens firstly fractured in the condition of liquid nitrogen, and then etched away calcium carbonate from the fracture surface using hydrochloric acid. Therefore, the cavities shown in Fig. 6 represented calcium carbonate infills, in other words, Fig. 6 clearly depicted the dispersion of calcium carbonate infills in matrix, which indicated that the infill phase uniformly disperse in PE matrix processed by $\mathrm{S}^{3} \mathrm{M}$ pan-milling equipment.

Fig. 7 showed three optical images taken by ultra-depth three-dimensional microscope. Looked the samples in high magnification under the glare, the components morphology could be clearly observed on account of different components have different reflectivity. The images indicated that the size of dispersed phases turned smaller and the dispersibilities of dispersed phases turned better with the increasing of $\mathrm{S}^{3} \mathrm{M}$ cycles. The reasons was attributed to pan-milling mechanochemistry equipment providing very strong 3-dimentional shear forces for pulverizing and milling thermoplastic artificial grass fibers and carpet backing, thermoset adhesive and inorganic infills integrally into fine powders, in the period of milling, the size of components turned smaller and various kinds of powders mixed together.

Noticeably, phase morphology, as a determining factor for properties of multicomponent polymer systems, is controlled by the interfacial interactions and compatibility of the blends' components. Dynamic mechanical analysis (DMA) is widely and generally used to characterize compatibility of multicomponent polymer, and the value of $\tan \delta$ is closely related to the motion segment which is the most appropriate verdict on the compatibility. It should be underlined that $\tan \delta$ could characterize the compatibility though the following two methods: judging from the relations between $\alpha$ relaxation peaks of constituents which are corresponded with their own glass transition temperature $\left(T_{\mathrm{g}}\right)$ and the changes of peak values, peak intensity and peak width of $\tan \delta .^{22}$

Fig. 8 showed the $\tan \delta$ versus temperature curves of r-WAT via different milling cycles, all five curves have similar profiles, two visible relaxation peaks can be seen at around $T=$ $25{ }^{\circ} \mathrm{C}$ and $T=90{ }^{\circ} \mathrm{C}$ through the temperature monitored. As mentioned above, the main components of WAT are PE and PP. PET is rarely exist in the WAT. Although the $T_{\mathrm{g}}$ of PP and PET are $25{ }^{\circ} \mathrm{C}$ (ref. 23) and $85{ }^{\circ} \mathrm{C},{ }^{24}$ respectively, the two visible relaxation peaks in Fig. 8 were mainly contributed to the motion segment 


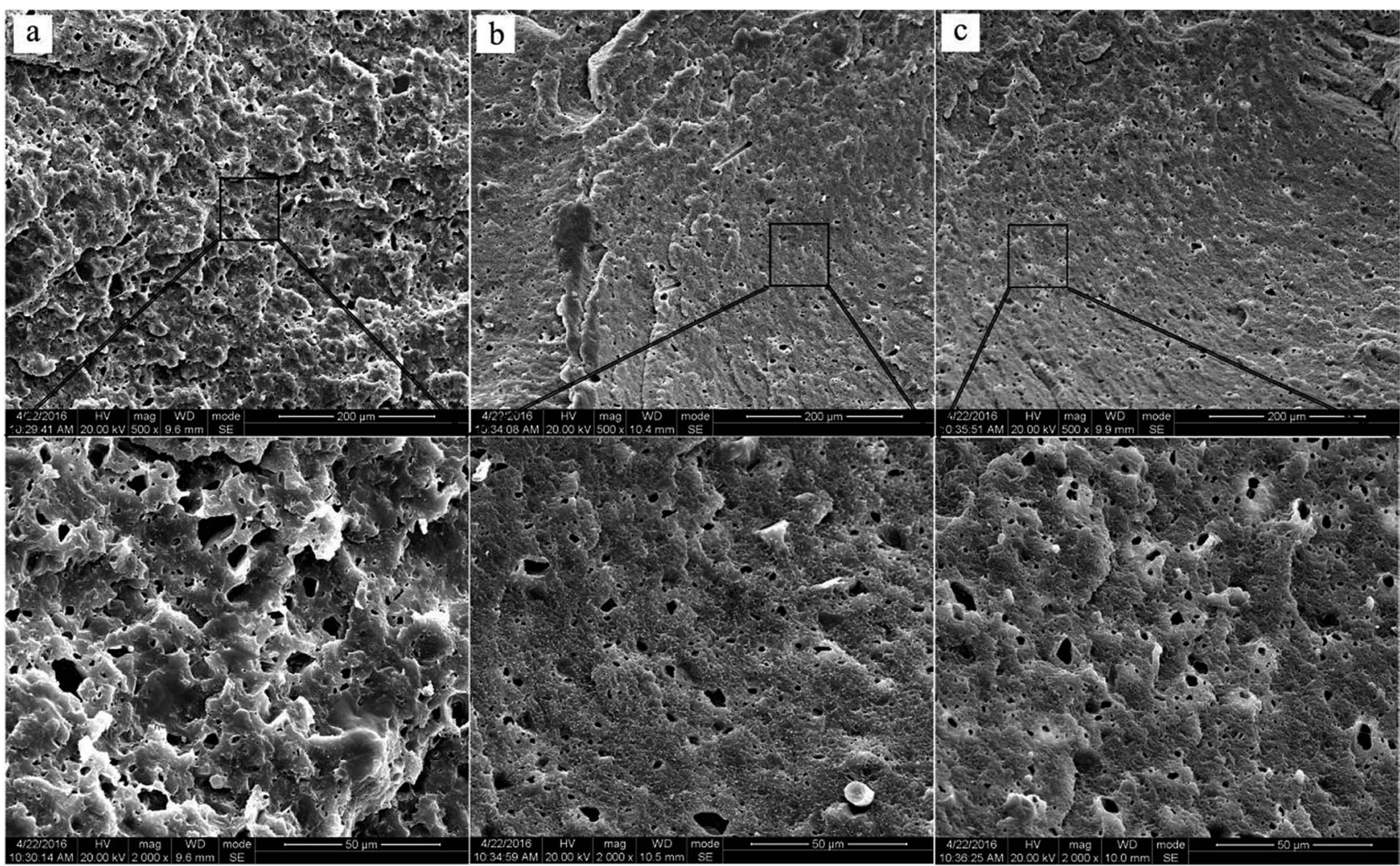

Fig. 6 SEM of the liquid-nitrogen-cryofractured surface of r-WATC via different cycles of S³: (a) 0 cycle; (b) 10 cycles; (c) 20 cycles.

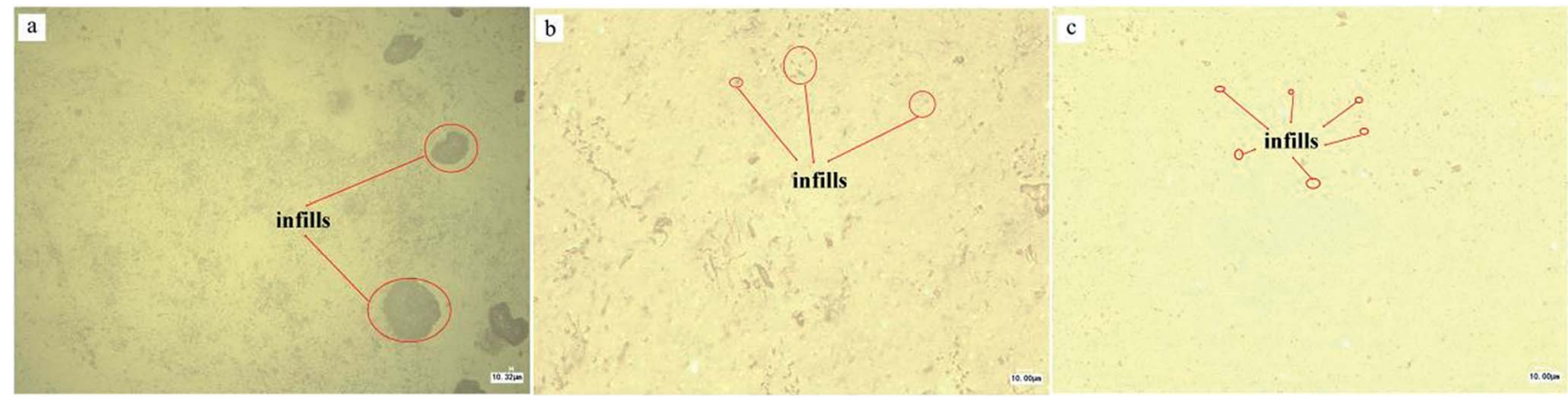

Fig. 7 Optical images of $r$-WATC via different cycles of $S^{3} M$ by ultra-depth three-dimensional microscope: (a) 0 cycle; (b) 10 cycles; (c) 20 cycles.

of PE crystalline region, and the relaxation peak at lower temperature is $\beta$-relaxation which has been concluded that this transition results from the relaxation of chain units which are located in the interfacial region, and that at higher temperature is $\alpha$-relaxation which have clearly been verified ${ }^{25,26}$ that it is due to motions of chain units which lie within the crystalline portion of the polymer.

It can be observed from Fig. 8, peak values increased substantially with increasing milling cycles, raising from $32{ }^{\circ} \mathrm{C}$ to $40{ }^{\circ} \mathrm{C}$, and additionally half peak width widened apparently which showed that the motion of molecular lying in crystalline portion of PE motion is blocked, the reason of this result may be attributed to the molecular migration of other phases into interfacial region of PE crystalline portion, astricting molecular motion. Moreover, the peak intensity was increased with the milling cycles increased, indicating that the amount of migratory molecular grows in number, which can be explained that the more intensive of the peak intensity, the higher internal friction of polymer segment motion will be.

For those results, it was concluded that the $S^{3} \mathrm{M}$ technology could improve the compatibility of this multicomponent blends, WAT.

\subsection{X-ray diffraction analysis}

X-ray diffraction is widely used to study the structure, orientation, and size of the crystallite and crystallinity. 


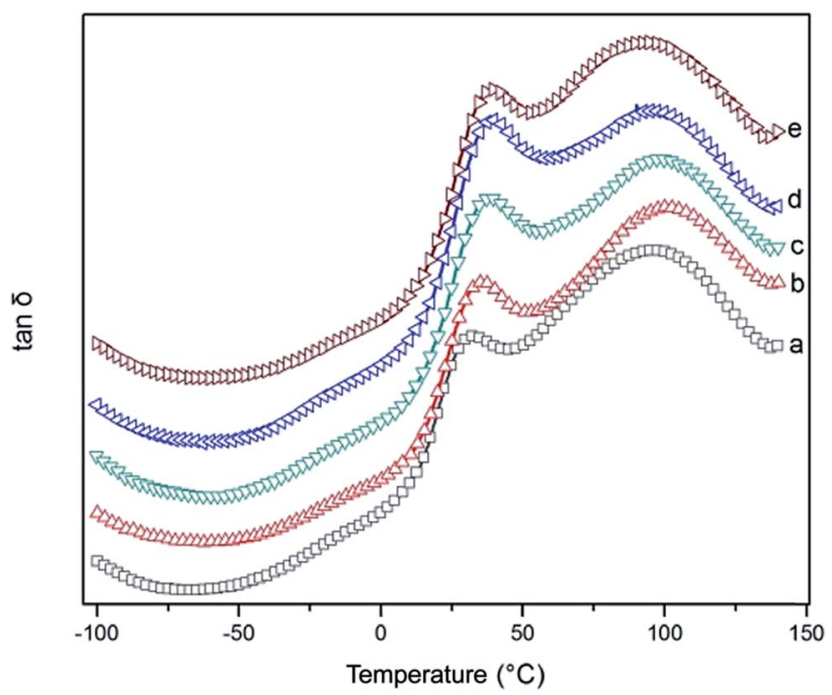

Fig. 8 Loss tangent versus temperature of $r$-WATC via different $S^{3} \mathrm{M}$ cycles: (a) 0 cycle; (b) 5 cycles; (c) 10 cycles; (d) 15 cycles; (e) 20 cycles.

Fig. 9 showed the X-ray diffraction curves of r-WAT milled 0 cycle, 5 cycles, 10 cycles, 15 cycles, 20 cycles, respectively. All five curves have similar profiles, a visible crystal diffraction peak can be seen at around $2 \theta=21.5^{\circ}$, and a broad crystal diffraction peak can be seen at around $2 \theta=23.5^{\circ}$. Besides, as it shown in Fig. 9, the diffraction peaks of pure PE were observed at $2 \theta$ Bragg's angles of $21.6^{\circ}$ and $24.0^{\circ}$ which correspond to diffraction planes of 110 and 200, generally in accordance with the curves of r-WAT. While the diffraction peaks of pure PP appeared at $2 \theta$ of $14.0^{\circ}, 16.8^{\circ}, 18.4^{\circ}, 21.0^{\circ}$, and $21.7^{\circ}$ which correspond to diffraction planes of the $110,044,130,131$, and 111, respectively, in marked contrast to the curves of r-WAT. So the two crystal diffraction peaks of r-WAT were contributed to the PE crystalline phase. Also, a scattering peak appearing at around $2 \theta=19.5^{\circ}$ stands for the amorphous portion.

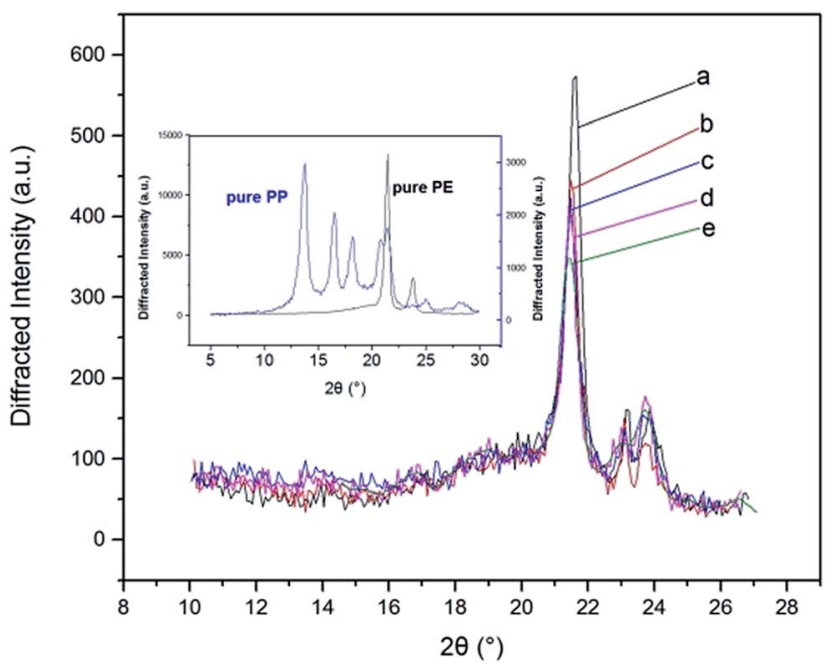

Fig. 9 X-ray diffraction patterns of pure PP, pure PE and $r$-WATC via different $\mathrm{S}^{3} \mathrm{M}$ cycles: (a) 0 cycle; (b) 5 cycles; (c) 10 cycles; (d) 15 cycles; (e) 20 cycles.
It was clearly noted from the Fig. 9 that the peak intensity of diffraction curves of r-WAT turned weak with the increase of milling cycles, indicating that milling and grinding hugely affect the crystalline ability of PE. And quantificationally, the values of the crystallite $\operatorname{size}^{27}$ and the crystallinity could be calculated by the following eqn (1) and (2), respectively.

$$
L_{h k l}=\frac{K \lambda}{\beta \cos \theta}
$$

where $L_{h k l}$ is the crystallite size perpendicular to the ( $h k l$ ) plane, $\theta$ is the angle between the incidence X-ray and the plane perpendicular to the $(h k l)$ plane, $K$ is the crystallite shape factor, 0.89 here, $\lambda$ is the wavelength of $\mathrm{X}$-ray, $0.1542 \mathrm{~nm}$ here, $\beta$ is the width of the diffraction peak at its half height or full width half maximum (FWHM).

$$
W_{\mathrm{c}, \mathrm{x}}=\frac{I_{\mathrm{c}}}{I_{\mathrm{c}}+K_{\mathrm{x}} I_{\mathrm{a}}}
$$

where $W_{\mathrm{c}, \mathrm{x}}$ is the degree of crystallinity; $I_{\mathrm{c}}$ and $I_{\mathrm{a}}$ is the diffraction integral intensity of crystalline region and amorphous region, respectively, in the appropriate angle range; $K_{\mathrm{x}}$ is the correction factor, 1 here. And the diffraction integral intensity could be replaced by the diffraction peak integral area.

The crystallite parameters were list in Table 1 , in which it was clearly observed that by increasing milling cycles, the crystallite size decreased, more specifically, the crystallite size of 110 crystal plane dropping from $25.65 \mathrm{~nm}$ to $23.30 \mathrm{~nm}$ and 200 crystal plane dropping from $26.08 \mathrm{~nm}$ to $14.74 \mathrm{~nm}$. Also, it was noted that the crystallite size increased slightly after milling 10 cycles and 15 cycles. The reasons of this behavior may be attributed to agglomeration of powders in the period of milling. Generally, the $\mathrm{S}^{3} \mathrm{M}$ could minished the crystallite size. The panmilling equipment can act as 3-dimentional scissors, providing very strong shear forces for pulverizing, dispersing and mixing WAL blends, making other polymer segmers migrate into PE phase, which broke the regularity of PE segmers. The destruction of segmers regularity made the crystallite size of sphaero crystal decrease. Besides, the $\mathrm{S}^{3} \mathrm{M}$ decreased the size of calcium

Table 1 Crystallite parameters obtained from the XRD curves analysis

\begin{tabular}{llllll}
\hline $\mathrm{S}^{3} \mathrm{M}$ cycles & $2 \theta /^{\circ}$ & $\beta /^{\circ}$ & $L_{\text {hkl }} / \mathrm{nm}$ & Area & $W_{\mathrm{c}, \mathrm{x}} / \%$ \\
\hline \multirow{2}{*}{0 cycle } & 21.610 & 0.613 & 25.65 & 2419 & 57.11 \\
& 23.92 & 0.603 & 26.08 & 360 & \\
& 19.53 & - & - & 2087 & \\
5 cycles & 21.59 & 0.621 & 25.32 & 2306 & 64.92 \\
& 23.92 & 0.648 & 24.27 & 363 & \\
& 19.71 & - & - & 2283 & \\
10 cycles & 21.50 & 0.677 & 23.23 & 1644 & 58.37 \\
& 23.84 & 1.087 & 14.47 & 710 & \\
\multirow{3}{*}{15 cycles } & 19.25 & - & - & 1722 & \\
& 21.47 & 0.627 & 25.08 & 1510 & \\
& 23.83 & 1.190 & 13.22 & 991 & 59.77 \\
20 cycles & 19.12 & - & - & 1683 & \\
& 21.34 & 0.675 & 23.30 & 2500 & \\
& 23.70 & 1.067 & 1405 & 1405 & 51.35 \\
& 19.28 & - & - & 3699 &
\end{tabular}


carbonate inorganic infills, the fine calcium carbonate powders played as a role of nucleating agent, promoting the segmers forming microcrystal.

The behavior of other polymer segmers migrated into $\mathrm{PE}$ phase hindered segmers motion in different extent, affecting the rate of segments regularly diffused, migrated and arranged during crystallization. That accounted for the reducing of degree of crystallinity, shown in Table 1.

In general, the $\mathrm{S}^{3} \mathrm{M}$ technology made the crystallite size decrease, and the smaller crystallite size could effectively improve the mechanical properties. Besides, the reducing of crystallinity supported the DMA conclusion that the molecular migration of other phases into PE crystalline portion after milling, and the compatibilities were improved.

Fig. 10 described the schematic illustration of $\mathrm{S}^{3} \mathrm{M}$ technology milling WAT, the strong 3-dimensional shear forces of $\mathrm{S}^{3} \mathrm{M}$ technology ultrafine milled WAT at the room temperature, making its structure be totally destroyed and all ingredients turn into indiscriminate ultrafine powder and randomly disperse, in which the domain size and the compatibility of the material were controlled by the particle size and its distribution of the composite powder at the solid state.

3.3.1 Processing property. The choice of suitable processing conditions is guided mainly by the rheological behavior of composites, so an investigation of rheological properties of molten polymers is fundamentally and practically importance.

Fig. 11 showed the effect of milling cycles on the MFI. From this figure it was distinctly noticed that when beginning milling, the MFI was slightly higher than that of without milling, besides as the milling cycles continued to increase, the MFI increased substantially, jumping from $2.491 \mathrm{~g} / 10 \mathrm{~min}$ to $4.0875 \mathrm{~g} / 10 \mathrm{~min}$, which illustrated that $S^{3} \mathrm{M}$ technology could effectively improve flowability.

Fig. 12 depicted that the curves of shear viscosity of r-WAT milled 0 cycle, 10 cycles, 20 cycles, respectively, versus shear

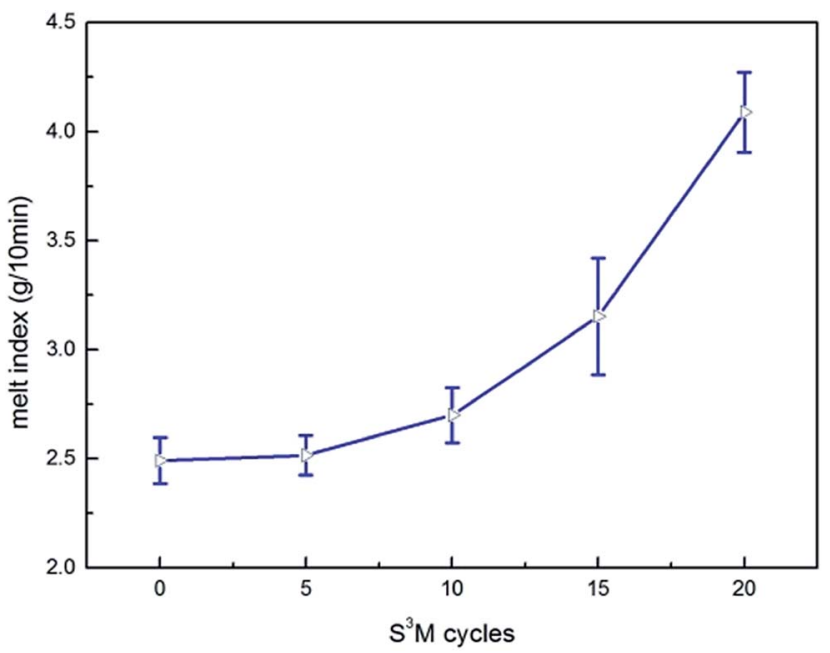

Fig. 11 The curves of melt flow index versus $S^{3} M$ cycles.

rate. Obviously, the WAT melts behaved as pseudoplastic, which was typical non-Newtonian fluids, in other words, the apparent viscosity of r-WAT reduced with the sequentially increase of shear rate. From the Fig. 12, it was also noticed that the apparent viscosity of r-WAT disposed by $\mathrm{S}^{3} \mathrm{M}$ was lower than that of without milling in the same shear rate. It is indicated that the $S^{3} \mathrm{M}$ could reduce the apparent viscosity of this multicomponent blends to a certain extent.

So it is concluded from rheological analysis that the $S^{3} \mathrm{M}$ technology could effectively improve the flowability and reduced the apparent viscosity of WAT melts.

It should be noticed that the apparent viscosity of WAT without milling reduced faster than that disposed by $\mathrm{S}^{3} \mathrm{M}$ with the shear rate sequentially increased from Fig. 12, it could be reasonably infer from this tendency that the apparent viscosity of WAT without milling would be lower than that disposed by

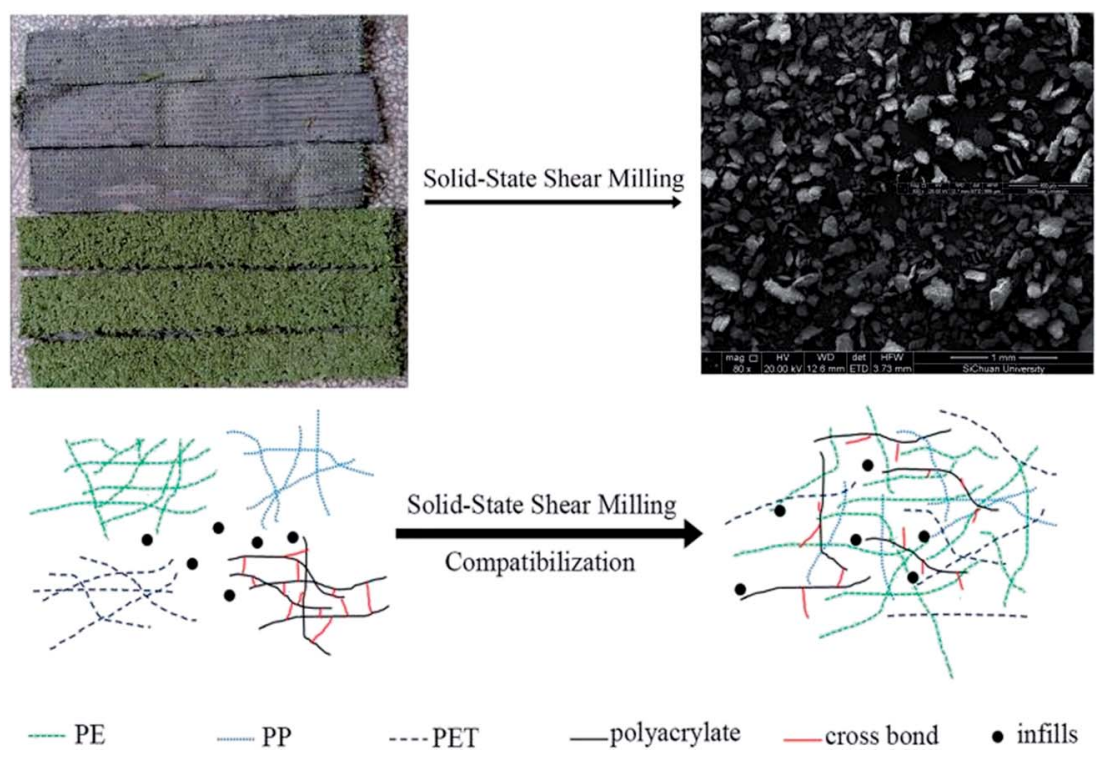

Fig. 10 Schematic illustration of $S^{3} M$ technology milling WAT. 


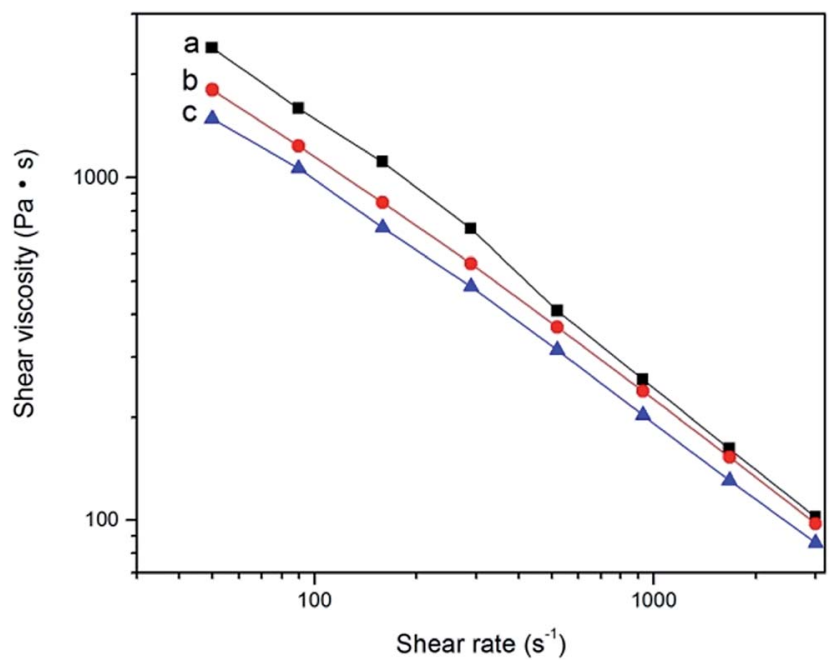

Fig. 12 The curves of apparent viscosity of $r$-WATC via $S^{3} \mathrm{M}$ versus shear rate: (a) 0 cycle; (b) 10 cycles; (c) 20 cycles.

$\mathrm{S}^{3} \mathrm{M}$ in higher shear rate which would lead to unsteady-state flow of WAT melts, arising the phenomena of melt fracture.

Fig. 13 depicted the surface appearance of the extrudater without milling which looked rough and uneven, displaying the phenomena of melt fracture, on the contrary, extrudate with 10 cycles milling looked smooth and glossy, and the surface appearance of extrudater milled 20 cycles got more smooth and glossier.

The reason for displaying the phenomena of melt fracture is that the melts flowing in the tube slipped and the spring back in melts. In the period of the melts flowed in the tube, the shear rate near tube was fastest and the distribution was uneven, making the distribution of elastic energy in the melts along the radial direction existed differences, this uneven distribution of elastic energy would generate elastic stress in the directions roughly paralleling to the velocity gradient. Once the elastic stress rose to the value of viscous flow resistance, the balance of the elastic stress in the melts would be broke, consequently the spring back happened. The uneven distribution of the complex components in PE matrix would undoubtedly result in uneven distribution of elastic energy. $\mathrm{S}^{3} \mathrm{M}$ could efficaciously improve the distribution, consequently the surface appearance of extrudater disposed by $\mathrm{S}^{3} \mathrm{M}$ looked smooth, and yet the surface appearance of extrudater without milling looked rough.

From those above results, it was concluded that $\mathrm{S}^{3} \mathrm{M}$ technology could efficaciously recycle waste artificial turf and improve and ameliorate processability. The reason of this behavior was attributed to that $\mathrm{S}^{3} \mathrm{M}$ technology could control the composite powder particle size and particle size distribution, and make the incompatible and complex components blend even and interpenetrating.

3.3.2 Mechanical properties. Fig. 14 showed curves of stress versus strain for r-WAT milled 0 cycle, 5 cycles, 10 cycles, 15 cycles, 20 cycles, respectively. This figure substantially and sufficiently provided that the tensile strength and elongation at break improved distinctly after disposed by $\mathrm{S}^{3} \mathrm{M}$. When beginning milling, the tensile strength and elongation at break were evidently higher than that of without milling, besides as the milling cycles continued to increase, the tensile strength and

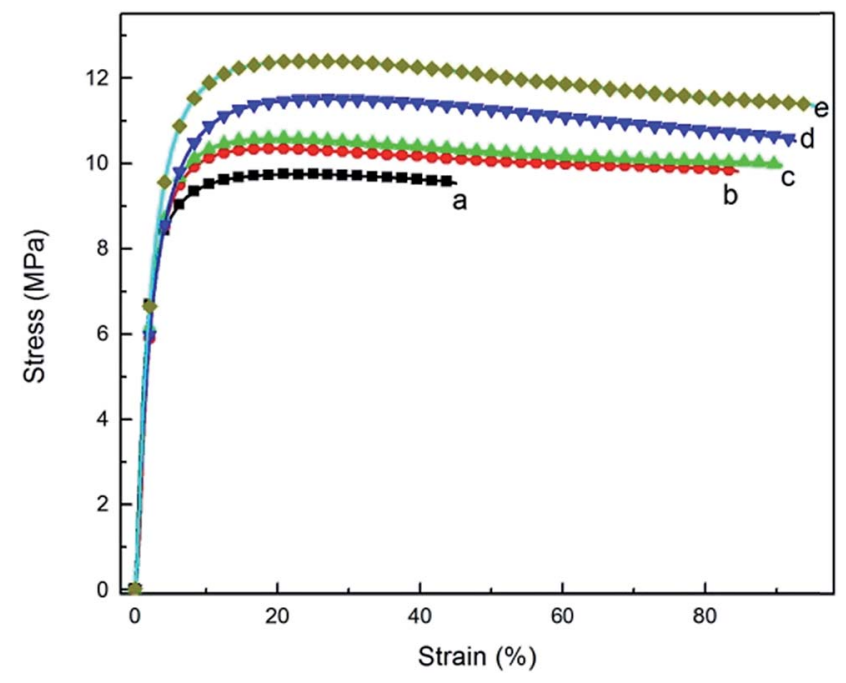

Fig. 14 Stress versus strain curves for $r$-WATC via different $S^{3} M$ cycles: (a) 0 cycle; (b) 5 cycles; (c) 10 cycles; (d) 15 cycles; (e) 20 cycles.

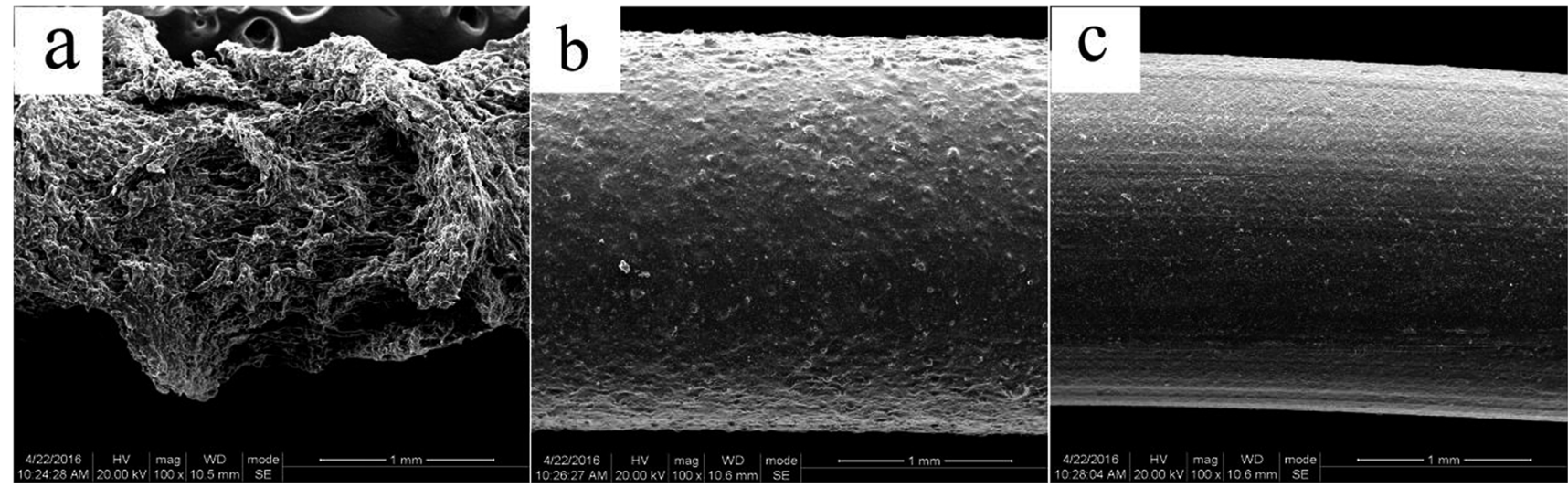

Fig. 13 SEM of $r$-WATC extrudate via different cycles of $S^{3} M$ : (a) 0 cycle; (b) 10 cycles; (c) 20 cycles. 

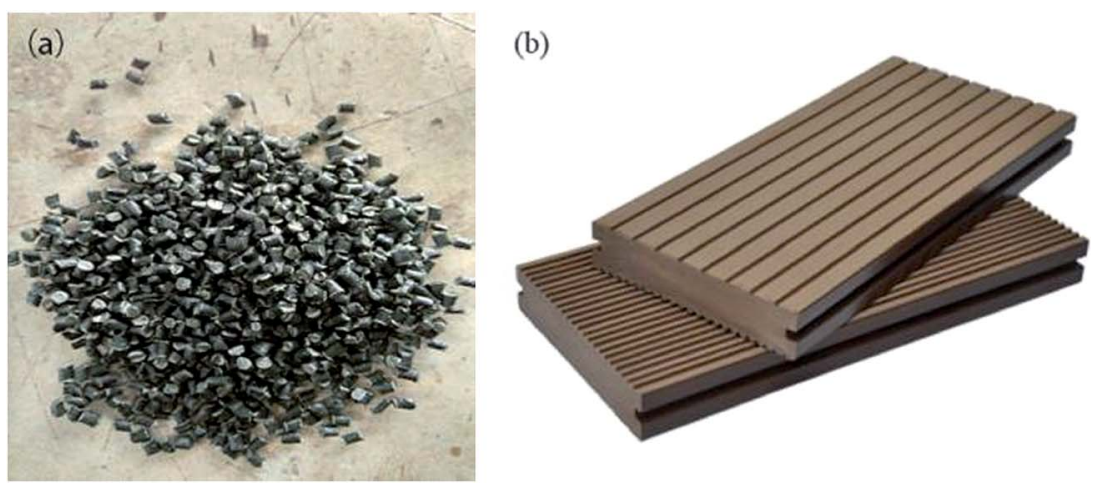

Fig. 15 The images of WAT pellets (a) and wood/plastics products (b).

Table 2 The properties of wood/plastics composites

\begin{tabular}{llll}
\hline & HDPE/WAT/WPs & HDPE/WPs & National standard \\
\hline Flexural strength (MPa) & 18.8 & 20.38 & $\geq 16.0$ \\
Flexural modulus (MPa) & 2040 & 1496 & $\geq 1800$ \\
Shore hardness (HD) & 69 & 66 & $\geq 55$ \\
Cost (¥ per t) & 2900 & 4600 & -
\end{tabular}

elongation at break improved persistently, increasing from 9.63 $\mathrm{MPa}$ to $12.64 \mathrm{MPa}$, from $47.67 \%$ to $96.29 \%$ respectively. From this result, it was concluded that the $S^{3} \mathrm{M}$ could improve strengths and tenacity of r-WAT. The reason of this behavior may be attributed to $S^{3} M$ effect. It is well known that the compatibilities and distributions of complex multicomponent blends and crystallographic structures of the crystalline portion make the great influence on the mechanical properties. In this work, SEM and ultra-depth three-dimensional (3D) microscope analysis have been used to characterize the phase morphology of multicomponent WAT, DMA has been used to characterize the compatibilities of r-WAT and XRD has been used to characterize the crystallinity and crystallite size of $\mathrm{PE}$ crystal phase. The results demonstrated that as $\mathrm{S}^{3} \mathrm{M}$ cycles increased, the dispersity and compatibility of r-WAT multicomponent improved and the crystallite size of main phase decreased. Generally, $\mathrm{S}^{3} \mathrm{M}$ technology successfully controlled the composite powder particle size and particle size distribution and minished the phase size, improving the performance of products.

\subsection{Application and economic evaluation}

The WAT fine powders have been successfully obtained from WAT fragments by $\mathrm{S}^{3} \mathrm{M}$ technology, and it has been used to prepare WAT pellets, shown in Fig. 15a, with $12.64 \mathrm{MPa}$ in tensile strength and $96.29 \%$ in elongation at break. These WAT pellets can be used to produce value-added products such as wood/plastics products, geomembranes, bellows and geogrids, showed in Fig. 15b.

In this work, WAT pellets have been successfully used to replace part of polyethylene materials to prepare wood/plastics products. HDPE blended with WAT pellets and WPs to prepare wood/plastics composites whose properties were recorded in table. From Table 2, it can be found that the properties of these products mingling with WAT pellets were basically equivalent to those of the pure material and generally meet the national standard, GB/T 24137-2009.

\section{Conclusions}

The $S^{3} \mathrm{M}$ technique milled WAT into indiscriminate ultrafine powder at room temperature successfully and effectively, and the domain size and the performance of the material were controlled by the particle size and its distribution of the composite powder at the solid state. The domain size of the material became smaller with the increase of milling circle. The $S^{3} M$ technology improved the compatibility between the components and effectively decreased crystallite size of PE in WAT, and thermal processability of the recycling material turned better after $\mathrm{S}^{3} \mathrm{M}$ milling. The tensile strength and the elongation at break of the recycling material can reach 12.6 MPa and $96.3 \%$, respectively. Moreover, the wood-plastics products made from WAT fine powders can be produced with considerable economic benefits and social effects. Thus, solid-state shear milling can act as a new method for the high-value recycling of WAT.

\section{Conflicts of interest}

There are no conflicts to declare.

\section{Acknowledgements}

This project was supported by the National Natural Science Foundation of China (51421061) and the Program of Innovative 
Research Team for Young Scientists of Sichuan Province (2016TD0010).

\section{References}

1 Eunomia Research \& Consulting Ltd, https://footballtechnology.fifa.com/media/1230/artificial_turf_recycling.pdf, 2007.

2 B. Fordyce, http://www.landscapeonline.com/research/ article.php/14635, 2011.

3 H. Cheng, Y. Hu and M. Reinhard, Environ. Sci. Technol., 2014, 48, 2114-2129.

4 O. Eriksson, M. Carlsson Reich, B. Frostell, A. Björklund, G. Assefa, J. O. Sundqvist, J. Granath, A. Baky and L. Thyselius, J. Cleaner Prod., 2005, 13, 241-252.

5 B. Gworek, W. Dmuchowski, E. Koda, M. Marecka, A. Baczewska, P. Brągoszewska, A. Sieczka and P. Osiński, Water, 2016, 8, 470.

6 S. Kaoser, S. Barrington and M. Elektorowicz, J. Soil Contam., 2010, 9, 503-522.

7 P. Usapein and O. Chavalparit, J. Mater. Cycles Waste Manage., 2013, 16, 373-383.

8 P. He, S. Bai and Q. Wang, Composites, Part B, 2016, 99, 373380.

9 P. Wei and S. Bai, RSC Adv., 2015, 5, 93697-93705.

10 S. Yang, S. Bai and Q. Wang, Waste Manage., 2016, 57, 168.

11 S. Yang, S. Bai and Q. Wang, J. Appl. Polym. Sci., 2015, 132, 3225-3232.

12 Q. Wang, J. Cao, J. Huang and X. Xu, Polym. Eng. Sci., 1997, 37, 1091-1101.
13 J. Zhu, X. Zhang, M. Liang and C. Lu, J. Polym. Res., 2010, 18, 533-539.

14 W. Shao, Q. Wang, F. Wang and Y. Chen, Carbon, 2006, 44, 2708-2714.

15 G. Wang, Y. Chen and Q. Wang, J. Polym. Sci., Part B: Polym. Phys., 2008, 46, 807-817.

16 B. Zhu, D. Ma, H. Li, Z. Sun and J. Wang, J. Macromol. Sci., Part B: Phys., 2016, 55, 188-200.

17 M. Poletto, M. Zeni and A. J. Zattera, J. Thermoplast. Compos. Mater., 2011, 25, 821-833.

18 J. Tian, W. Yu and C. Zhou, Polymer, 2006, 47, 7962-7969.

19 M. Parvinzadeh, S. Moradian, A. Rashidi and M.-E. Yazdanshenas, Appl. Surf. Sci., 2010, 256, 2792-2802.

20 Y. Zhu, C. Liang, Y. Bo and S. Xu, J. Polym. Res., 2015, 22, 35.

21 S. L. Chai, M. M. Jin and H. M. Tan, Eur. Polym. J., 2008, 44, 3306-3313.

22 Q. Wu, N. Chen and Q. Wang, J. Polym. Res., 2010, 17, 903909.

23 P. K. Mandal and D. Chakraborty, J. Appl. Polym. Sci., 2009, 111, 2345-2352.

24 Y. Zhang, H. Zhang, Y. Yu, W. Guo and C. Wu, J. Appl. Polym. Sci., 2009, 114, 1187-1194.

25 G. Karine, P. Vinicius and S. L. Cristine, J. Appl. Polym. Sci., 2016, 133, 42887-42892.

26 R. Popli, M. Glotin and L. Mandelkern, J. Polym. Sci., Polym. Phys. Ed., 1984, 22, 407-449.

27 A. L. Patterson, Phys. Rev., 1939, 56, 978-982. 